\title{
Choline-Functionalized Supramolecular Copolymers: Toward Antimicrobial Activity against Streptococcus pneumoniae
}

\author{
Marle E. J. Vleugels, Silvia Varela-Aramburu, Bas F. M. de Waal, Sandra M. C. Schoenmakers, \\ Beatriz Maestro, Anja R. A. Palmans, Jesús M. Sanz,* and E. W. Meijer*
}

Cite This: Biomacromolecules 2021, 22, 5363-5373

Read Online

\section{ACCESS \\ 山ll Metrics \& More \\ Article Recommendations \\ Supporting Information}

ABSTRACT: Dynamic binding events are key to arrive at functionality in nature, and these events are often governed by electrostatic or hydrophobic interactions. Synthetic supramolecular polymers are promising candidates to obtain biomaterials that mimic this dynamicity. Here, we created four new functional monomers based on the benzene-1,3,5-tricarboxamide (BTA) motif. Choline or atropine groups were introduced to obtain functional monomers capable of competing with the cell wall of Streptococcus pneumoniae for binding of essential choline-binding

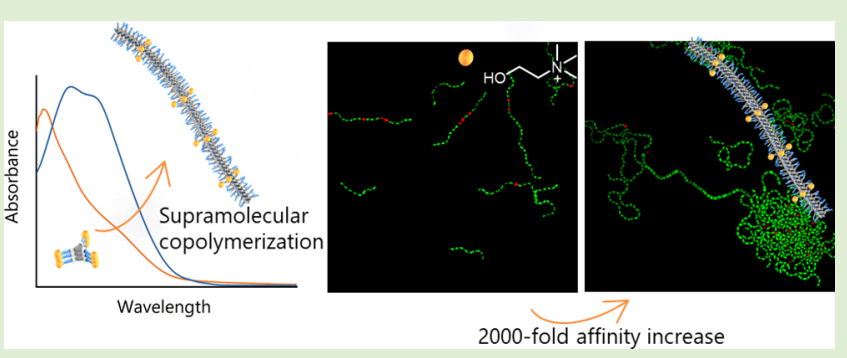
proteins (CBPs). Atropine-functionalized monomers BTA-Atr and

BTA-Atr ${ }_{3}$ were too hydrophobic to form homogeneous assemblies, while choline-functionalized monomers BTA-Chol and BTA$\mathbf{C h o l}_{3}$ were unable to form fibers due to charge repulsion. However, copolymerization of BTA-Chol ${ }_{3}$ with non-functionalized BTA$(\mathbf{O H})_{3}$ yielded dynamic fibers, similar to BTA- $(\mathbf{O H})_{3}$. These copolymers showed an increased affinity toward CBPs compared to free choline due to multivalent effects. BTA-based supramolecular copolymers are therefore a versatile platform to design bioactive and dynamic supramolecular polymers with novel biotechnological properties.

\section{INTRODUCTION}

Supramolecular polymers are ubiquitous in nature and vital for biological systems. ${ }^{1}$ Amyloid fibrils, for example, are highly organized fiber structures with a high stability and dynamicity. These fibrils are widely present in natural systems and they are associated with diseases in humans as well as with survival of bacteria in host organisms. ${ }^{2,3}$ To mimic these highly stable but dynamic fibers and also to expand functionality and modularity, synthetic supramolecular polymers forming 1D fibers are of interest. ${ }^{4,5}$ Peptide amphiphiles, consisting of a hydrophobic hydrocarbon chain conjugated to a peptide block, can form $1 \mathrm{D}$ nanostructures in water driven by $\beta$-sheet formation and hydrophobic interactions. ${ }^{6}$ Depending on the bioactive components present in the peptide block, several functionalities have been achieved for bone regeneration or stabilization of growth factors. ${ }^{7,8}$ For ureido-pyrimidinone (UPy)-based supramolecular polymers, dimerization of the UPy moieties combined with stacking of the urea groups can lead to $1 \mathrm{D}$ fiber formation in water by the combined effects of hydrogen bonding, hydrophobic interactions, and $\pi$-stacking. ${ }^{9,10}$ In this system, functionality can be introduced by the conjugation of active molecules to the UPy moiety and co-assembling these functional monomers with the UPy polymer. In solution, the incorporation of, for example, peptides or charged moieties within the UPy assemblies resulted in growth factor stabilization or siRNA delivery. ${ }^{11,12}$

Another well-studied supramolecular motif is benzene-1,3,5tricarboxamide (BTA), which can lead to fiber formation in water due to a combination of hydrophobic interactions, $\pi$ stacking, and threefold hydrogen bonding. ${ }^{13,14}$ An alkyl spacer of 11 or 12 carbons in combination with tetraethylene glycol is required to achieve the correct hydrophobic-hydrophilic balance for the protection of the inner hydrogen bonds while also having enough hydrophilicity to form $1 \mathrm{D}$ fibers in water. ${ }^{14} \mathrm{~A}$ recent detailed study showed that these $1 \mathrm{D}$ fibers are actually double helices with a half-pitch length between 15 and $25 \mathrm{~nm}^{15}$ An alkyl spacer of 12 carbons has been shown to form the best reproducible assemblies in water and has thus been studied most extensively. ${ }^{16}$ BTA-based supramolecular polymers in water are dynamic, as monomers are constantly being exchanged with the aqueous environment and between different polymers. ${ }^{16,17}$ Upon addition of ssDNA as an anionic multivalent recruiter, cationic monomers are capable of dynamically rearranging and clustering along the fiber, similar to raft formation in cell membranes. ${ }^{18}$ Several functionalities such as peptides and small carbohydrates have also been introduced within BTA

Received: September 29, 2021

Revised: November 12, 2021

Published: November 30, 2021

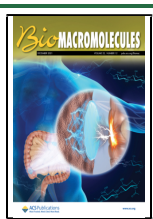


copolymers. ${ }^{17,19-21}$ Recently, the interaction between benzoxaborole-functionalized BTA fibers and human red blood cells was studied, showing that the fibers are capable of binding to sialic acid residues present on the surface of these cells. Moreover, only a low number of functional monomers within the assembly are needed for binding, hinting at the role of multivalency in the cell/material interaction. ${ }^{22}$

To further explore BTA-based supramolecular polymers in biomedical applications, the interactions with pathogens are of special interest. In general, monovalent interactions between pathogens and single binding partners are weak, but a multivalent presentation of the binding partners can substantially increase the binding affinity. ${ }^{23}$ In this scenario, BTA fibers show potential as a long and dynamic multivalent platform for pathogen binding for therapeutic or diagnostic applications. To explore this, the Gram-positive bacterium Streptococcus pneumoniae (pneumococcus) was chosen as a target microorganism as it is an important respiratory pathogen worldwide, a major agent causing bacterial pneumonia and meningitis and one of the main bacterial co-infectants with the SARS-CoV2 virus. ${ }^{24}$ In addition, antibiotic resistance issues are being increasingly reported in recent years, with a high incidence in the pneumococcal case, ${ }^{25}$ thus creating the need for new paradigms to develop novel antimicrobial agents. ${ }^{26,27}$ S. pneumoniae possesses a cholinedecorated cell wall able to specifically bind the surface cholinebinding proteins (CBPs). These CBPs play essential roles in the bacterial life cycle, such as the separation of daughter cells upon division, adhesion to the host, and toxin release. ${ }^{28}$ Choline and choline analogues such as atropine are known to bind to CBPs and thereby inhibit the binding of CBP cell-wall hydrolases to the cell surface, impeding the daughter cells' separation upon division and resulting in the formation of long bacterial chains. ${ }^{29-31}$ These chains are presumed to have lower infectivity and to be more prone to phagocytosis. ${ }^{32-34}$ While the affinity of these compounds for CBPs is too low for effective therapeutic use (dissociation constants in the millimolar range), our previous work showed that a multivalent presentation of choline and atropine on dendrimer scaffolds exponentially increased the binding affinity toward CBPs. ${ }^{35,36}$

Here, the question arises whether a multivalent presentation of CBP inhibitors such as choline or atropine on a modular and dynamic BTA-based supramolecular polymer could give rise to an increase in binding affinity compared to its static, dendrimeric counterpart. To this end, we designed a set of four new monomers based on the previously reported water-soluble BTA$(\mathrm{OH})_{3}$ monomers functionalized with one or three choline/ atropine substituents. ${ }^{13}$ The assembly of the functional monomers (BTA-Chol, BTA-Chol ${ }_{3}$, BTA-Atr, and BTA$\mathrm{Atr}_{3}$ ) was studied as homo-assemblies and as copolymers with BTA- $(\mathbf{O H})_{3}$ in water using spectroscopic and light-scattering techniques as well as cryogenic transmission electron microscopy (cryo-TEM). The dynamicity of the supramolecular copolymers was investigated with hydrogen/deuterium exchange followed by mass spectrometry (HDX-MS). Finally, the binding of these supramolecular polymers toward CBPs was examined and compared with free choline and cholinecontaining dendrimers. The exogenous addition of BTA$\mathrm{Chol}_{3} /$ BTA- $(\mathrm{OH})_{3}$ copolymers on pneumococcal planktonic cultures induced drastic phenotypic changes in the bacteria (cell chaining and aggregation), demonstrating multivalent effects of 3 orders of magnitude and paving the way for their biomedical use as efficient theranostic agents.

\section{EXPERIMENTAL SECTION}

Materials. Unless stated otherwise, all reagents and chemicals were obtained from commercial sources at the highest purity available and used without further purification. All solvents were of AR quality and purchased from Biosolve. Water was purified on an EMD Millipore Milli-Q Integral Water Purification System. Reactions were followed by thin-layer chromatography (precoated $0.25 \mathrm{~mm}, 60-\mathrm{F} 254$ silica gel plates from Merck). Dry solvents were obtained with an MBRAUN Solvent Purification System (MB-SPS). Ion exchange resin DOWEX 1X8-50 (Cl-form) was obtained from Acros. Prior to use, a column was first washed with demineralized water, followed by washing with methanol. Weakly basic resin Amberlite IRA-95 (Aldrich) was washed with water, methanol, and again water before use. Automated column chromatography was performed on a Biotage Isolera using Biotage SNAP-KP SIL cartridges. $\mathrm{H}_{2} \mathrm{~N}-\mathrm{C}_{12}-\mathrm{EO}_{4}-\mathrm{N}_{3}{ }^{18} \mathrm{H}_{2} \mathrm{~N}-\mathrm{C}_{12}-\mathrm{EO}_{4}-\mathrm{OBn},{ }^{13}$ Chol-NHS, ${ }^{35}$ Atr- $\mathrm{C}_{5}-\mathrm{OH}^{*} \mathrm{HCl}^{36}{ }^{3}$ DMT-MM, ${ }^{37}$ 5-methoxycarbonylbenzene-1,3-dicarboxylic acid (3), ${ }^{38}$ and BTA- $(\mathrm{OH})_{3}{ }^{13}$ were synthesized according to previously published procedures.

Synthesis of the Monomers. All details of the synthesis, purification, and characterization of the four new monomers are given in the SI: BTA-Chol ${ }_{3}$ (ESI-MS: calculated $\mathrm{m} / z=1675.23$, observed $\mathrm{m} / z=558.83[\mathrm{M}]^{3+}$ ), BTA-Atr 3 (MALDI-ToF-MS: calculated $\mathrm{MW}=2400.27 \mathrm{~g} / \mathrm{mol}$, observed $\mathrm{m} / \mathrm{z}=2400.68[\mathrm{M}+$ $\left.\mathrm{H}]^{+}, 2422.65[\mathrm{M}+\mathrm{Na}]^{+}\right)$, BTA-Chol (ESI-MS: calculated $\mathrm{m} / z=$ 1417.03, observed $\left.m / z=1417.08[\mathrm{M}]^{+}, 709.25[\mathrm{M}+\mathrm{H}]^{2+}\right)$, and BTAAtr (MALDI-ToF: calculated MW $=1659.29$, observed $m / z=1660.18$ $\left.[\mathrm{M}+\mathrm{H}]^{+}, 1682.16[\mathrm{M}+\mathrm{Na}]^{+}\right)$.

Assembly of BTA Materials. Homo-assembly: BTA- $(\mathrm{OH})_{3}$, BTA-Atr ${ }_{3}$, BTA-Chol $_{3}$, BTA-Chol, and BTA-Chol ${ }_{3}$ samples were prepared by weighing the solid material into a glass vial, adding Milli-Q (MQ) water to obtain the desired concentration, stirring the sample at $80{ }^{\circ} \mathrm{C}$ for $15 \mathrm{~min}$, and vortexing the sample for $15 \mathrm{~s}$ immediately afterward. All samples were left to equilibrate overnight at room temperature before being used for any measurements. Co-assembly: BTA- $(\mathrm{OH})_{3}$ was co-assembled with functional monomers by weighing BTA- $(\mathrm{OH})_{3}$ as a solid material into a glass vial and adding BTA-Chol or BTA-Chol ${ }_{3}$ from a $500 \mu \mathrm{M}$ stock solution in $\mathrm{MeOH}$. The organic solvent was removed using an $\mathrm{N}_{2}(\mathrm{~g})$ stream after which MQ water was added to obtain the desired concentration. The sample was stirred at 80 ${ }^{\circ} \mathrm{C}$ for $15 \mathrm{~min}$ and vortexed for $15 \mathrm{~s}$ immediately afterward. All samples were left to equilibrate overnight at room temperature before being used for any measurements.

Analytical Techniques. ${ }^{1} \mathrm{H}$ NMR and ${ }^{13} \mathrm{C}$ NMR spectra were recorded on a Varian Mercury Vx $400 \mathrm{MHz}\left(400 \mathrm{MHz}\right.$ for ${ }^{1} \mathrm{H}$ NMR and $100 \mathrm{MHz}$ for ${ }^{13} \mathrm{C}$ NMR). Proton chemical shifts are reported in parts per million (ppm, $\delta$ ) downfield from trimethylsilane (TMS) using the resonance frequency of the deuterated solvent as the internal standard. Peak multiplicity is abbreviated as s: singlet; d: doublet, q: quartet; p: pentet; m: multiplet; dd: double doublet; dt: double triplet; dq: double quartet; carbon chemical shifts are reported in $\mathrm{ppm}(\delta)$ downfield from TMS using the resonance frequency of the deuterated solvent as the internal standard.

Matrix-assisted laser absorption/ionization mass time of flight (MALDI-TOF) spectra were obtained on a Bruker Autoflex Speed. $\alpha$-Cyano-4-hydroxycinnamic acid and trans-2-[3-(4-tert-butylphenyl)2-methyl-2-propenylidene]malononitrile were used as matrixes.

Liquid chromatography mass spectrometry (LC-MS) was performed on a system consisting of the following components: a Shimadzu SCL-10A VP system controller with Shimadzu LC-10AD VP liquid chromatography pumps with an Alltima C18 $3 \mathrm{u}(50 \times 2.1 \mathrm{~mm})$ reversed-phase column and gradients of water-acetonitrile supplemented with $0.1 \%$ formic acid, a Shimadzu DGU 20A3 prominence degasser, a Thermo Finnigan surveyor auto sampler, a Thermo Finnigan surveyor PDA detector, and a Thermo Scientific LCQ Fleet. Gradients were run from $5 \% \mathrm{MeCN}$ to $100 \% \mathrm{MeCN}$ over a $15 \mathrm{~m}$ period.

Ultraviolet-visible (UV-vis) absorbance spectra were recorded on a JASCO V-650 UV-vis spectrometer or a JASCO V-750 UV-vis spectrometer with a JASCO ETCT-762 temperature controller. 
Measurements were performed using Quartz cuvettes with a pathlength of $1 \mathrm{~mm}$ (500 $\mu \mathrm{M}$ samples) or $1 \mathrm{~cm}$ (50 $\mu \mathrm{M}$ samples). First, a baseline of the corresponding solvent was measured. All measurements were performed with a bandwidth of $1.0 \mathrm{~nm}$, a scan speed of $100 \mathrm{~nm} / \mathrm{min}$, and a data interval of $0.1 \mathrm{~nm}$, spanning the UV-vis range from 350 to $190 \mathrm{~nm}$. All spectra were averaged over three measurements.

Static light scattering (SLS) measurements were performed on an ALV ALVCGS-3 Compact Goniometer equipped with an ALV5000 digital correlator and a HeNe laser operating at $532 \mathrm{~nm}$. The scattering intensity was detected over the angular range of $30-150^{\circ}$ with steps of $5^{\circ}$, with 10 runs of $10 \mathrm{~s}$ per angle. BTA samples were prepared at a concentration of 500 or $250 \mu \mathrm{M}$ and were measured in light scattering tubes with an outer diameter of $1 \mathrm{~cm}$. As a reference, samples of only the corresponding solvent and only toluene were measured. Water was filtered with a $0.2 \mu \mathrm{m}$ syringe filter (Supor membrane, PALL Corporation), and toluene was filtered with a $0.2 \mu \mathrm{m}$ syringe filter (PTFE membrane, Whatman). The measurements were analyzed with AfterALV (1.0d, Dullware) to remove measurements showing obvious scattering from dust. The Rayleigh ratio as a function of the angle was computed using the equation below with toluene as a reference

$$
R_{\theta}=\frac{I_{\text {sample }}-I_{\text {solvent }}}{I_{\text {toluene }}} * R_{\text {toluene }} * \frac{n_{\text {solvent }}^{2}}{n_{\text {toluene }}^{2}}
$$

with $I_{\text {sample }}$ the count rate of the sample solution, $I_{\text {solvent }}$ the count rate for the solvent (water), and $I_{\text {toluene }}$ the count rate for toluene. $R_{\text {toluene }}$ is the known Rayleigh ratio of toluene $\left(2.1 \times 10^{-2} \mathrm{~m}^{-1}\right.$ at $\left.532 \mathrm{~nm}\right), n_{\text {solvent }}$ is the refractive index of the solvent ( 1.333 for water), and $n_{\text {toluene }}$ is the refractive index of toluene (1.497).

HDX-MS measurements were carried out using a XevoTM G2 QTof mass spectrometer (Waters) with a capillary voltage of $2.7 \mathrm{kV}$, a sampling cone voltage of $20 \mathrm{~V}$, and an extraction cone voltage of $4.0 \mathrm{~V}$. The source temperature was set at $100^{\circ} \mathrm{C}$, the desolvation temperature at $400{ }^{\circ} \mathrm{C}$, the cone gas flow at $10 \mathrm{~L} \mathrm{~h}^{-1}$, and the desolvation gas flow at $100 \mathrm{~L} \mathrm{~h}^{-1}$. The sample solutions subjected to HDX were introduced into the mass spectrometer using a Harvard syringe pump (11 Plus, Harvard Apparatus) at a flow rate of $50 \mu \mathrm{L} \mathrm{min}^{-1}$. Previously prepared BTA samples of $500 \mu \mathrm{M}$ in MQ water were diluted 10 times with $\mathrm{D}_{2} \mathrm{O}$ (including $0.5 \mathrm{mM}$ sodium acetate to facilitate detection), resulting in a final concentration of $50 \mu \mathrm{M}$. The samples were stored at room temperature during the experiment. MS spectra of supramolecular assemblies in water were recorded after several time points after dilution. For homo-assemblies, ACN was added to a sample diluted into $\mathrm{D}_{2} \mathrm{O}$ in a roughly $1: 1$ volume ratio to study the MS spectra of disassembled supramolecular systems.

Cryo-TEM was performed on samples with a concentration of 250 or $500 \mu \mathrm{M}$ of BTAs in water. Vitrified films were prepared in a "Vitrobot" instrument (FEI Vitrobot Mark IV, FEI Company) at $22{ }^{\circ} \mathrm{C}$ and at a relative humidity of $100 \%$. In the preparation chamber of the "Vitrobot", $3 \mu \mathrm{L}$ samples were applied on Quantifoil grids (R 2/2, Quantifoil Micro Tools GmbH) or Lacey grids (LC200-Cu, Electron Microscopy Sciences), which were surface plasma-treated just prior to use (Cressington 208 carbon coater operating at $5 \mathrm{~mA}$ for $40 \mathrm{~s}$ ). Excess sample was removed by blotting using filter paper for $3 \mathrm{~s}$ with a blotting force of -1 , and the thin film thus formed was plunged (acceleration of about $3 \mathrm{~g}$ ) into liquid ethane just above its freezing point. The vitrified films were transferred into the vacuum of a CryoTITAN equipped with a field emission gun that was operated at $300 \mathrm{kV}$, a post-column Gatan energy filter, and a $2048 \times 2048$ Gatan CCD camera. Vitrified films were observed using a CryoTITAN microscope at temperatures below $-170{ }^{\circ} \mathrm{C}$. Micrographs were taken at low dose conditions, starting at a magnification of 6500 with a defocus setting of $-40 \mu \mathrm{m}$ and at a magnification of 24,000 with a defocus setting of $-10 \mu \mathrm{m}$.

Expression and Purification of the C-LytA-GFP Protein. CLytA-GFP was purified by affinity chromatography in DEAE-cellulose from the overproducing Escherichia coli strain REG1 [pALEX2-CaGFP] as previously described. ${ }^{35,39}$ The eluted protein was extensively dialyzed against $10 \mathrm{mM} \mathrm{NH}_{4} \mathrm{HCO}_{3}$ and freeze-dried at $-80{ }^{\circ} \mathrm{C}$. The protein concentration was determined spectrophotometrically using a molar absorption coefficient at $280 \mathrm{~nm}\left(\varepsilon_{280}\right)$ of $11,1730 \mathrm{M}^{-1} \mathrm{~cm}^{-1}$ as predicted by ProtParam software (http://web.expasy.org/protparam).

Fluorescence anisotropy (FA) was performed using a Tecan Infinite M1000 plate reader $\left(\lambda_{\mathrm{ex}}: 485 \pm 20 \mathrm{~nm}, \lambda_{\mathrm{em}}: 535 \pm 25 \mathrm{~nm}\right.$, mirror: Dichroic 510, G-factor: 0.935 manual, flashes: 20, integration time: 50 $\mu \mathrm{s}$, settle time: $0 \mu \mathrm{s}$, gain: optimal, and Z-position: calculated from the well). $0.1 \mu \mathrm{M}$ C-LytA-GFP was incubated overnight at RT with BTA fibers (serial dilution starting from $250 \mu \mathrm{M}$ BTA, triplicate preparations). All FA measurements were conducted in phosphate buffer ( $20 \mathrm{mM}$ sodium phosphate buffer, $50 \mathrm{mM} \mathrm{NaCl}, \mathrm{pH} 7.0$ ) in polystyrene non-binding low-volume Corning Black Round Bottom 384-well plates (Corning 4514) in a final sample volume of $10 \mu \mathrm{L}$ per well.

Molecular Docking. The BTA-Chol ${ }_{3}$ monomer was generated with ChemDraw and subjected to energy minimization with Chem3D contained in the ChemOffice 17 utilities (PerkinElmer Informatics). Files with the coordinates of the BTA-Chol ${ }_{3}$ monomer and the C-LytA protein (PDB code $1 \mathrm{HCX})^{40}$ were then submitted to the SwissDock server (http://www.swissdock.ch/). ${ }^{41,42}$

Bacterial Strain and Growth Conditions. S. pneumoniae R6CIB17 is a non-flocculant, non-encapsulated strain derived from the capsular type- 2 clinical isolate strain D39. ${ }^{33,43}$ Liquid cultures were grown at $37{ }^{\circ} \mathrm{C}$ without aeration in $\mathrm{C}$ medium supplemented with $0.08 \%(\mathrm{w} / \mathrm{v})$ yeast extract $(\mathrm{C}+\mathrm{Y}$ medium $) .{ }^{44}$ Growth was monitored by measuring the optical density at $550 \mathrm{~nm}\left(\mathrm{OD}_{550}\right)$ in an Evolution 201 spectrophotometer (Thermo Scientific). Prior to every measurement, the tubes were normally turned over several times to homogenize the solution, except when the flocculating capacity of additives was being assayed. At an optical density of 0.1 , equal volumes of the corresponding stocks of additives (choline or BTA polymers) were added to the medium. For the control experiment, the same volume of phosphate buffer was added.

The number of viable cells was determined by counting the colonies that appeared from appropriate dilutions of the culture (in triplicate) after overnight incubation at $37^{\circ} \mathrm{C}$ on trypticase soy plates (CondaPronadisa) supplemented with $5 \%$ defibrinated sheep blood (Thermo Fisher).

Laser Scanning Confocal Microscopy. At different times of growth, a sample of the pneumococcal liquid culture was taken, and cells were stained with the LIVE/DEAD BacLight bacterial viability kit (Molecular Probes) to monitor the bacterial populations. Following the instructions of the manufacturer, a $3 \mu \mathrm{L}$ mixture of a 1:1 SYTO 9/ propidium iodide mix was added to a $1 \mathrm{~mL}$ sample of the culture medium and kept at room temperature for $15 \mathrm{~min}$ in the dark. The final concentrations of SYTO 9 and propidium iodide in the culture medium were 5 and $30 \mu \mathrm{M}$, respectively. Confocal images were captured using an inverse laser scanning confocal microscope (Leika TCS-SP2-AOBSUV) with a $63 \times$ oil-immersion lens and with a zoom $2 \times$. The excitation/emission wavelengths for SYTO 9 and propidium iodide were $488 / 500-550$ and 543/600-670 nm, respectively.

\section{RESULTS AND DISCUSSION}

Molecular Design and Synthesis. The monomers were designed based on the water-soluble BTA- $(\mathbf{O H})_{3}$, which is known to form micrometer-long fibers in water. ${ }^{13}$ Choline and atropine were introduced at the periphery of the ethylene glycol moiety to ensure proper presentation and availability of the functional groups for binding upon (co)polymerization of the functional monomers. To investigate the effect of the number of charged or hydrophobic groups within functional monomers on supramolecular (co)polymerization, the functional monomers bore either one (BTA-Chol and BTA-Atr) or three (BTA$\mathrm{Chol}_{3}$ and BTA-Atr ${ }_{3}$ ) functional groups at their outer periphery (Figure 1).

All functional monomers were obtained via amine-modified BTA intermediates. For the triple-substituted monomers, a symmetric amine-modified BTA core (BTA- $\left.\left(\mathbf{N H}_{2}\right)_{3}\right)$ was obtained by the reaction of 1,3,5-tricarbonyl trichloride with 


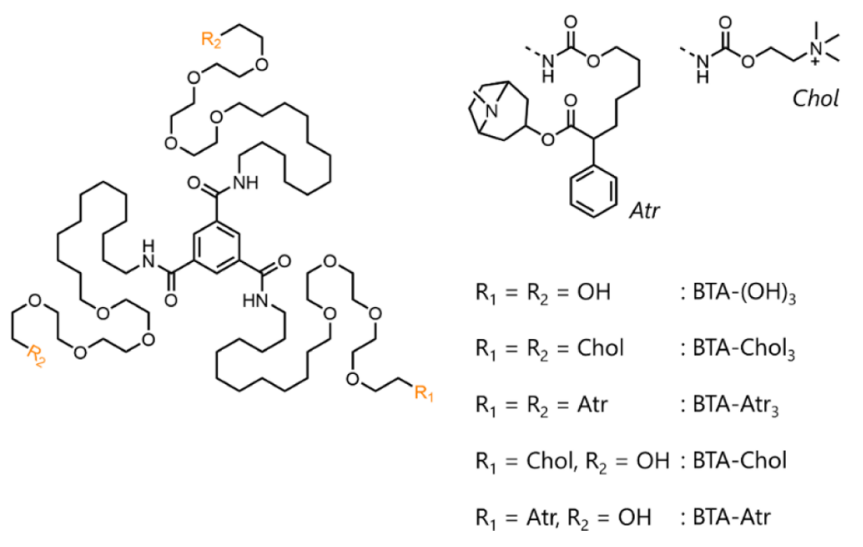

Figure 1. Chemical structures of the BTA monomers used in this study.

azide-modified side chains (Scheme S1), followed by reduction with triphenylphosphine. Further coupling of BTA- $\left(\mathbf{N H}_{2}\right)_{3}$ with the activated ester of choline yielded BTA- $\mathbf{C h o l}_{3}$. Atropinebased monomer BTA-Atr ${ }_{3}$ was obtained via activation of the atropine hydroxyl linker with 4-nitrophenyl carbonate and subsequent coupling with BTA- $\left(\mathbf{N H}_{2}\right)_{3}$ (Scheme 1$)$. The asymmetric BTA core BTA- $\mathbf{N H}_{2}$ was obtained starting from the desymmetrized trimesic acid monomethyl ester (Scheme S2) and consecutive activation, coupling, and deprotection steps. BTA- $\mathbf{N H}_{2}$ was further reacted with activated choline and atropine linkers to obtain BTA-Chol and BTA-Atr (Scheme S2). The purity and the assignment of the BTA structures were confirmed using ${ }^{1} \mathrm{H}$ NMR, ${ }^{13} \mathrm{C}$ NMR, MALDI-TOF-MS, and LC-MS (Figures S1-S12 in the Supporting Information).

Supramolecular Homo-Assembly of the Functional Monomers. First, the homo-assemblies of the functionalized monomers in water were studied. The formation of supramolecular fibers by BTA- $(\mathbf{O H})_{3}$ was fully characterized previously, which revealed that the fibers possess two distinct absorption maxima in the UV-vis spectrum at 211 and $226 \mathrm{~nm}$ (Figure 2A). ${ }^{13}$ In contrast, both BTA-Chol and BTA-Chol ${ }_{3}$ show a single maximum at $194 \mathrm{~nm}$ (Figure 2A). This single band at a shorter wavelength is indicative of the formation of smaller, micellar-like aggregates. ${ }^{45}$ The presence of such smaller, micellar aggregates was corroborated with SLS (Figure 2B). In contrast to BTA- $(\mathbf{O H})_{3}$, which shows an angular dependency of the
Rayleigh ratio typical for elongated fibrillar structures, both BTA-Chol and BTA-Chol ${ }_{3}$ show a larger angular dependency, indicating a different type of morphology. The slightly lower Rayleigh ratio also indicates the presence of smaller assemblies compared to BTA- $(\mathrm{OH})_{3}$. Finally, visualization of these structures with cryo-TEM (Figure S13) further corroborates the formation of small assemblies. Thus, the introduction of choline into BTA monomers prevents the stacking into fibers most probably due to charge repulsion between the choline units. The amount of charge presented on the monomer does not greatly influence the formed assemblies as neither BTAChol nor BTA-Chol ${ }_{3}$ is capable of forming supramolecular fibers.

The atropine-functionalized monomers BTA-Atr and BTA$\mathbf{A t r}_{3}$ were then investigated as more hydrophobic alternatives for obtaining functionalized assemblies. However, due to a significantly decreased solubility in water, their assemblies were studied at a lower concentration than their choline counterpart. Again, in contrast to BTA- $(\mathbf{O H})_{3}$, for these assemblies, the typical absorption maxima in UV-vis for supramolecular fibers were absent (Figure 2C). Instead, for BTA-Atr, a shift in absorption to a lower wavelength is visible, while BTA-Atr ${ }_{3}$ shows scattering at a higher wavelength, indicative of an inhomogeneous solution caused by precipitates. As atropine has an absorbance spectrum in the same lower wavelength region (Figure S14), further interpretation by UVvis was complicated. On the other hand, SLS (Figure 2B) of BTA-Atr shows an angular dependency similar to the cholinefunctionalized BTAs, indicating that BTA-Atr does not form supramolecular fibers either. Visualization of the structures with cryo-TEM (Figure S15) shows only small assemblies for BTA$\mathbf{A t r}_{3}$ and unspecific cluster formation for BTA-Atr. The introduction of a hydrophobic group to BTA monomers thus prevents the stacking into fibers and decreases the water solubility.

To obtain fibrillar-like supramolecular polymers in water, we explored the incorporation of the functionalized monomers into supramolecular copolymers with BTA- $(\mathrm{OH})_{3}$. As atropinefunctionalized monomers showed decreased solubility in water, only the choline-functionalized monomers were further studied for their ability to be incorporated into supramolecular stacks.

Scheme 1. Synthetic Route toward Triple-Functionalized BTAs ${ }^{a}$
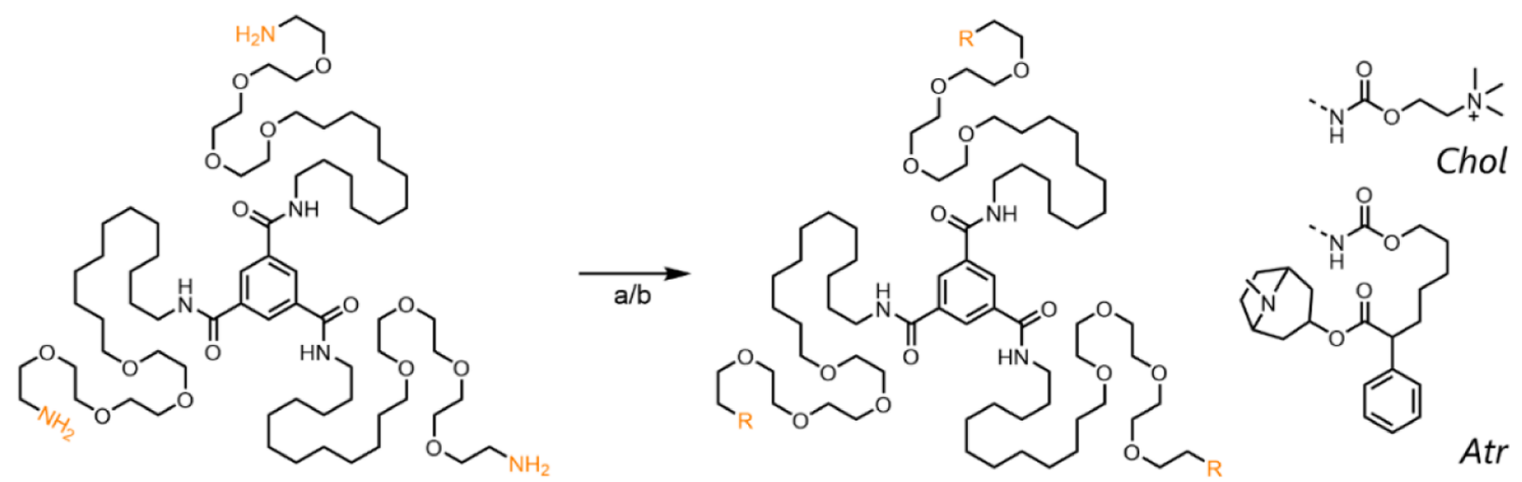

BTA- $\left(\mathrm{NH}_{2}\right)_{3}$

\footnotetext{
${ }^{a}$ Triple-functionalized BTA- $\mathbf{C h o l}_{3}$ and BTA-Atr ${ }_{3}$ were obtained via symmetric BTA- $\left(\mathrm{NH}_{2}\right)_{3}$. Mono-functionalized BTA-Chol and BTA-Atr were obtained via asymmetric BTA- $\mathrm{NH}_{2}$ (Scheme S2). (a) Chol-NHS, TEA, THF/ $\mathrm{H}_{2} \mathrm{O}, 39 \%$. (b) Atr- $\mathrm{C}_{5}-\mathrm{OH}^{*} \mathrm{HCl}$, 4-nitrophenyl carbonate, DIPEA, DMF, $64 \%$.
} 
(A)

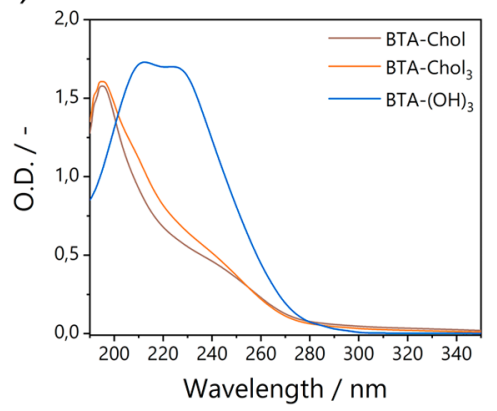

(B)

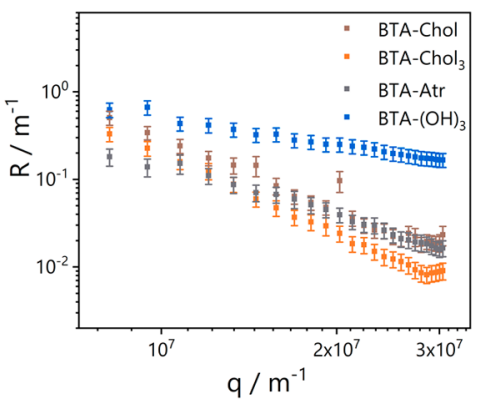

(C)

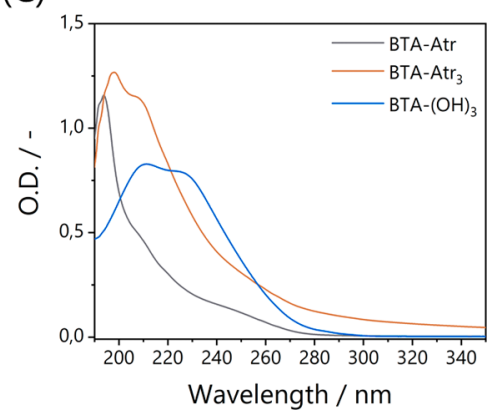

Figure 2. UV-vis (A,C) and SLS (B) spectra of homo-assemblies of functionalized BTA monomers in water $\left(c_{\mathcal{B}_{\mathrm{BA}}}=500 \mu \mathrm{M}\right.$ for BTA-OH${ }_{3}$, BTAChol, and BTA-Chol ${ }_{3}$ or $250 \mu \mathrm{M}$ for BTA-Atr and BTA-Atr $3, T=20^{\circ} \mathrm{C}$ ).

(A)

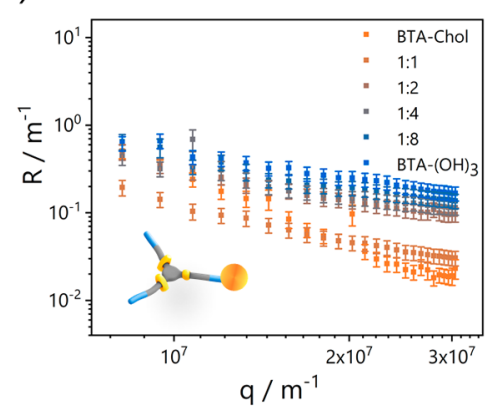

(D)

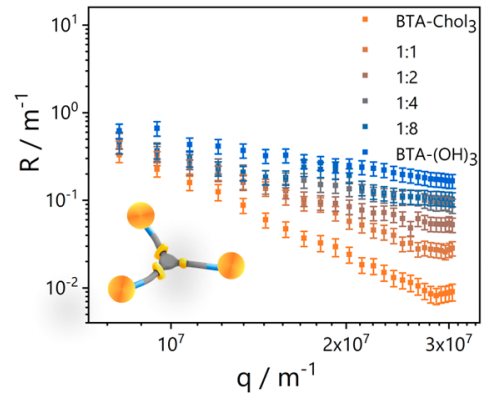

(B)

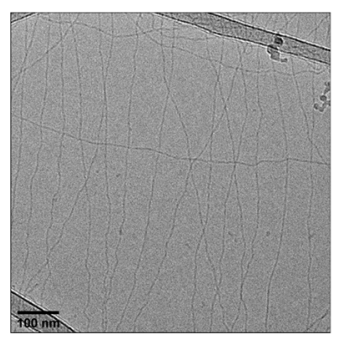

(E)

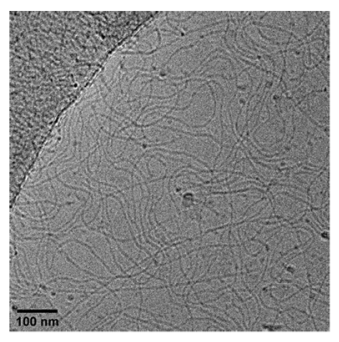

(C)

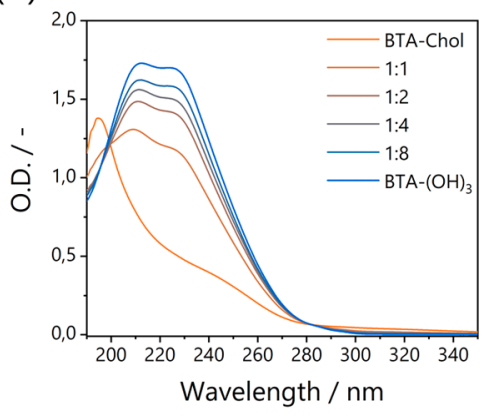

(F)

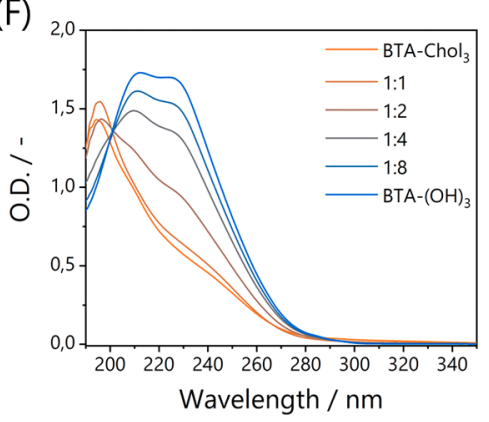

Figure 3. SLS (A,D), cryo-TEM images (B,D), and UV-vis (C,F) upon copolymerization of choline-functionalized monomers $\left(c_{\mathrm{BTA}}=500 \mu \mathrm{M}, \mathrm{T}=\right.$ $\left.20^{\circ} \mathrm{C}\right)$.

\section{Copolymerization of Choline-Functionalized Mono-}

mers. Supramolecular co-assemblies containing cholinefunctionalized monomers were prepared by mixing BTA$\mathrm{Chol}_{x}$ and BTA- $(\mathrm{OH})_{3}$ in molar ratios of 1:1 to 1:8 BTA$\mathrm{Chol}_{x} / \mathrm{BTA}-(\mathrm{OH})_{3}$. Investigation of the copolymerization of BTA-Chol using SLS (Figure 3A) shows a change in the slope and intensity with increasing amounts of BTA- $(\mathrm{OH})_{3}$ toward a scattering profile resembling BTA- $(\mathrm{OH})_{3}$. This indicates that the structures change from small aggregates into long fibrillar structures. Cryo-TEM of the BTA-Chol 1:8 copolymer (Figures $3 \mathrm{~B}$ and $\mathrm{S} 16 \mathrm{~A}$ ) confirms the formation of long fibrous structures. UV-vis spectroscopy (Figure 3C) shows the shift from a single band at a lower wavelength toward the typical absorption maxima at 211 and $226 \mathrm{~nm}$ from a 1:2 ratio, indicating that at this ratio, the functional monomers are already taken up into the stack.

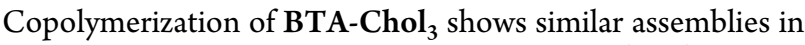
terms of size and morphology compared to BTA- $(\mathrm{OH})_{3}$ for the BTA-Chol ${ }_{3}$ 1:8 copolymer (Figure 3D). Cryo-TEM imaging of BTA-Chol $_{3}$ 1:8 (Figures $3 \mathrm{E}$ and S16B) corroborates the formation of long fibrous structures. However, fibers of different lengths can be seen for the BTA-Chol $\mathbf{~}_{3} \mathbf{1 : 2}$ co-assembly (Figure S17), confirming that upon high incorporation of monomers with a high charge density, a mixture of short and long assemblies is formed instead of co-assembly into long fibrous structures. UV-vis spectroscopy shows the gradual shift from a single band at a lower wavelength toward absorption maxima at 211 and $226 \mathrm{~nm}$ by increasing the ratio of BTA- $(\mathrm{OH})_{3}$ (Figure $3 \mathrm{~F})$, but a higher ratio of at least 1:8 BTA- $(\mathrm{OH})_{3}$ is needed to fully remove the shoulder at lower wavelength compared to copolymers with BTA-Chol. This higher ratio indicates that due to the higher charge present on the functional monomer, more repulsive interactions occur, and thus less $\mathbf{B T A}-\mathrm{Chol}_{3}$ can be incorporated as a co-monomer into the supramolecular stack.

Thus, upon copolymerization with BTA- $(\mathrm{OH})_{3}$, it is possible to obtain choline-functionalized supramolecular fibers although the maximum incorporation ratio is dependent on the number of charges in the monomer. To gain further insight into the incorporation of charged monomers into supramolecular polymers as well as the dynamics of the monomers within the supramolecular copolymers, HDX-MS was employed as a labelfree method previously used in our group. ${ }^{16,46,47}$ The three 
BTAs used in this study have six exchangeable hydrogen atoms and the exchange of these hydrogen atoms to deuterium upon dilution into $\mathrm{D}_{2} \mathrm{O}$ can be followed with MS. The outer hydroxyl or amide groups exchange immediately upon dilution, but the inner amides only exchange upon monomer migration into the surrounding $\mathrm{D}_{2} \mathrm{O}$ as they are protected from $\mathrm{D}_{2} \mathrm{O}$ in the hydrophobic pocket of the supramolecular stack. The H/D exchange can thus be related to monomer dynamicity. Hereto, $500 \mu \mathrm{M}$ BTA solutions were prepared in MQwater and 10 times diluted into $\mathrm{D}_{2} \mathrm{O}$. Upon dilution, the nature of the aggregates did not change (Figure S18). After dilution, the ESI-MS spectra were recorded at several time intervals, and the mass increase of both BTA- $(\mathrm{OH})_{3}$ and BTA-Chol ${ }_{3}$ monomers was followed over time (Figure S19, Supporting Information Section 2 for details about calculations of percentages of the deuterated species). First, both BTA-Chol and BTA-Chol ${ }_{3}$ homoassemblies were studied to confirm the fast exchange dynamics, showing indeed only one isotope distribution that is immediately visible after dilution of an aqueous sample into $\mathrm{D}_{2} \mathrm{O}$. These results correspond to fully deuterated BTA6D, indicating that the core amides are not shielded from the solvent (Figure S20). ${ }^{21,45}$ After copolymerization of BTA-Chol B $_{3}$ with BTA- $(\mathrm{OH})_{3}$, upon dilution, analogues without all their amide hydrogen atoms immediately exchanged for BTA-Chol ${ }_{3}$ (BTA1D-BTA5D, Figure S19) are visible. This indicates that the functional monomers are protected from surrounding $\mathrm{D}_{2} \mathrm{O}$ and thus incorporated into supramolecular stacks with a hydrophobic pocket shielding the core amides. To compare different assemblies, the percentage of BTA6D was plotted over time, as this relates to fully deuterated monomers, and is thus a measure of monomer dynamicity. Upon copolymerization of BTA-Chol ${ }_{3}$ with BTA- $(\mathrm{OH})_{3}$, a slower monomer exchange of BTA-Chol ${ }_{3}$ monomers compared to the homo-assembly is observed (Figure 4A), suggesting successful copolymerization. Similar to the BTA- $(\mathbf{O H})_{3}$ homo-assembly, the BTA-Chol ${ }_{3}$ and BTA- $(\mathrm{OH})_{3}$ monomers in the 1:8 co-assembly first show a fast monomer exchange, which slows down over time (Figure 4A,B). The difference in exchange dynamics of BTA- $(\mathbf{O H})_{3}$ in the homo-assembly and the 1:8 copolymer also indicates successful copolymerization (Figure 4B).

Choline-functionalized monomers can thus be incorporated into supramolecular polymers that retain their dynamic behavior. To test the accessibility of functional groups after copolymerization and if dynamicity still allowed for binding, the capability of choline-functionalized supramolecular polymers to interact with the CBPs of $S$. pneumoniae was assessed.

Binding of the Choline-Binding Module C-LytA to BTA-Chol ${ }_{x} /$ BTA-OH ${ }_{3}$ Supramolecular Fibers. The cholinebinding module (CBM) of the LytA autolysin (C-LytA) is the best characterized member of the CBMs present in all pneumococcal CBPs. ${ }^{28,39,48}$ It contains four choline-binding sites per monomer, and the protein dimerizes through its $\mathrm{C}$ terminal hairpin upon interaction with the ligand. ${ }^{40}$ This allows the monitoring of choline binding by using a fusion protein with the green fluorescent protein (C-LytA-GFP) since the cholineinduced dimerization brings the GFP moieties of each monomer to close proximity, leading to fluorescence energy transfer phenomena and resulting in a decrease of FA. ${ }^{35}$ The titration curve with choline chloride presents a sigmoidal shape (Figure $5 \mathrm{~A}$, red), as previously described for a binding-dimerization coupled process. ${ }^{35}$ Titration of C-LytA-GFP with BTA fibers up to a $250 \mu \mathrm{M}$ total monomer concentration (Figure $5 \mathrm{~B}$ ) shows that BTA-Chol ${ }_{3}$ 1:9 was also able to induce a sigmoidal change
(A)

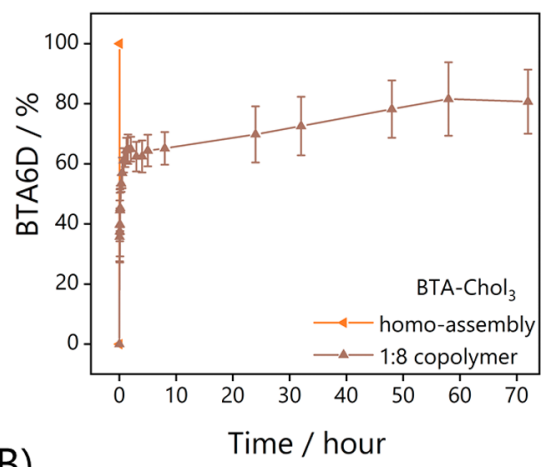

(B)

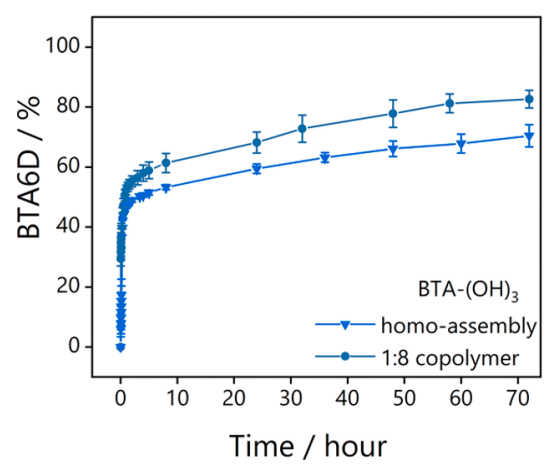

Figure 4. HDX-MS curves of (A) BTA-Chol ${ }_{3}$ and (B) BTA-(OH) monomers within homo-assemblies and copolymers of BTA-Chol ${ }_{3}$ and BTA- $(\mathrm{OH})_{3}$ after 10 times' dilution of an aqueous sample into $\mathrm{D}_{2} \mathrm{O}$. The graphs highlight the amount of fully exchanged monomers (BTA6D) as a function of time. Measurements were performed at room temperature; the error bars represent the standard deviation calculated from three separate experiments and lines are added to guide the eye (initial $c=500 \mu \mathrm{M}$; after dilution, $50 \mu \mathrm{M}$ ).

in anisotropy, while no change in the signal was detected with non-functionalized BTA- $(\mathrm{OH})_{3}$, suggesting that the interaction between the BTA-Chol ${ }_{3}$-containing fiber and the CBM is specific.

Furthermore, the titration half-point in choline concentration equivalents (Figure 5A, fit details in Supporting Information Section 3) was remarkably lower than the corresponding concentration of choline chloride, indicating a multivalent effect of nearly 2500 (Table 1) (calculation details in Supporting Information Section 4). This value is substantially higher than previously shown by a generation-5, choline-functionalized poly(propyleneimine) dendrimer (g5-cho) ${ }^{35}$ (around 400: Table 1), indicating that multivalent binding effects are more evident in the BTA fibers than in the smaller dendrimers. Remarkably, monosubstituted BTA-Chol 1:9 fibers were unable to show binding, similar to the control BTA- $(\mathbf{O H})_{3}$ (Figure 5B). As BTA-Chol 1:9 fibers display only one choline group per functional monomer, the concentrations were expressed in choline-equivalents to make a fair comparison between BTAChol 1:9 and BTA-Chol 1:9 (Figure 5A). Here, it is visible that BTA-Chol 1:9 is indeed not able to bind, even in the same

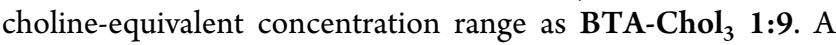
possible explanation for this difference could be the higher local concentration of choline on the triple-substituted monomers. Due to this higher concentration, a single BTA- $\mathbf{C h o l}_{3}$ monomer might simultaneously bind to two or three choline-binding sites in the C-LytA moiety, thus adding an additional multivalent 
(A)

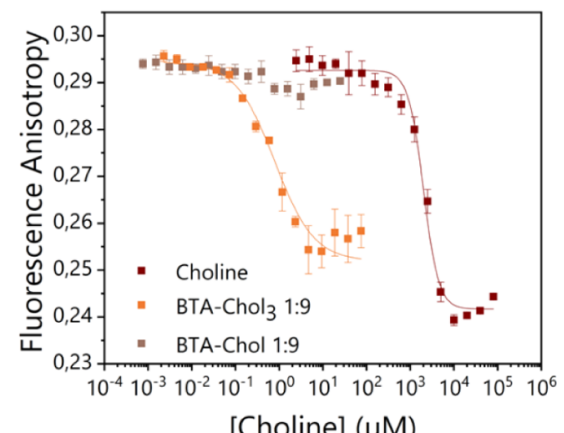

(B)

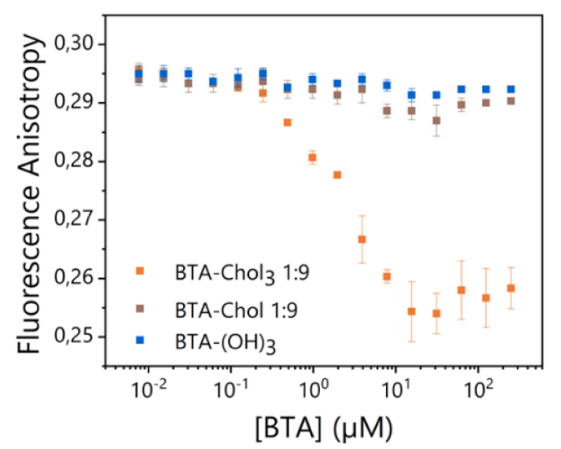

Figure 5. FA assays of C-LytA-GFP $(0.1 \mu \mathrm{M})$ (A) BTA fibers and choline chloride and (B) BTA fibers. Sigmoidal curves were fit with a logistic model.

feature that translates into a higher binding efficiency, contrary to monosubstituted BTA-Chol. To theoretically verify this possibility, we modeled the binding of a single BTA-Chol ${ }_{3}$ monomer to C-LytA with the SwissDock utilities. ${ }^{41,42}$ Figure 6 depicts one of the generated structures with the lowest energy, showing a BTA-Chol ${ }_{3}$ monomer fitting simultaneously to two binding sites through the quaternary ammonium group of the molecule.

Effect of BTA Fibers on Pneumococcal Cultures. After the confirmation of BTA-Chol ${ }_{3}$-functionalized fibers as efficient CBP-binding agents, the next step was to evaluate the addition of different functional BTA fibers to planktonic cultures of $S$. pneumoniae. CBP inhibition by exogenously added choline chloride leads to cancellation of autolysis and daughter cell separation, resulting in bacterial chain formation. ${ }^{29}$ Ideally, these effects should be observed upon incubation with BTA fibers as well but with lower concentrations needed due to multivalent presentation of choline on the supramolecular scaffold. To facilitate the observation of possible cell aggregates, the experiments were carried out with the R6CIB17 strain, a derivative of the R6 strain that does not flocculate in liquid media. 33
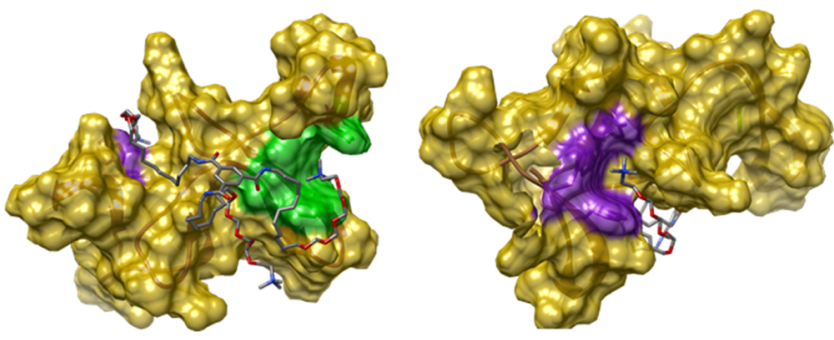

Figure 6. Two views of one of the lowest energy docking structures of a BTA-Chol $_{3}$ monomer on the C-LytA module, as generated by SwissDock. The choline-binding site configured by $\operatorname{Trp} 261, \operatorname{Trp} 268$, and Tyr293 is shown in green whereas that configured by Trp220, Trp268, and Trp293 is colored magenta.

Choline chloride or BTA fibers were added at an early exponential phase $\left(\mathrm{OD}_{550}=0.1\right)$, and bacterial growth was first monitored by turbidimetry. Figure S21A shows, for the R6CIB17 control, the typical pattern representative of an initial exponential phase, followed by a $\sim 3-4 \mathrm{~h}$ stationary phase and a final autolytic phase in which the LytA autolysin is massively released from the cytosolic pool, leading to a substantial decrease in optical density. ${ }^{49}$ In addition, Figure S21A shows the effect of the incubation of different concentrations of choline chloride. At $25 \mathrm{mM}$ and above, the autolytic phase no longer occurred as a consequence of the inhibition of LytA recognition of the cell wall due to saturation of its binding sites by the ligand, as previously described. ${ }^{29}$ Moreover, as other CBP cell wall hydrolases involved in cell separation upon division, such as LytB, were also inhibited, bacteria formed long chains instead of the typical diplococcal shape displayed by the control cells (Figure $7 \mathrm{~A}, \mathrm{~B}){ }^{29,50}$ Addition of BTA- $(\mathbf{O H})_{3}(50 \mu \mathrm{M})$ to the bacterial cultures led to visible aggregates (Figure S21C), but this did not exert any significant effect on the growth curve (Figure S21B). Analysis of these aggregates by confocal fluorescence microscopy (Figure 7C) showed that most of the cells remained green after BacLight staining, ruling out any detrimental effect of the BTA- $(\mathbf{O H})_{3}$ fibers on the bacterial membrane integrity. This was confirmed by bacterial viability assays as the viability was not altered compared to the control samples (Figure S22). Addition of BTA-Chol 1:9 $(50 \mu \mathrm{M})$ did not affect the growth curves either even after increasing the BTA concentration threefold to obtain similar choline equivalents as BTA-Chol $_{3}$ copolymers $(150 \mu \mathrm{M})$ (Figure S21B). However, the incorporation of positive charges in the copolymer reduced the number of bacterial aggregates (Figures S21C and 7D). Finally, BTA-Chol $_{3}$ 1:9 $(50 \mu \mathrm{M})$ induced the most appreciable changes in bacterial morphology as visible aggregates were further reduced (Figure S21C) and, most remarkably, clear cell chains were observed by microscopy (Figure $7 \mathrm{E}$ ). These cell chains were even of a longer length than those induced by choline (Figure 7B). The cell membrane was not compromised either as the vast majority of cells remained green-stained with BacLight (Figure $7 \mathrm{E}$ ) and were as viable as the non-treated bacteria

Table 1. Multivalent Effects of Choline-Containing Species on FA Titration and on Pneumococcal Planktonic Cultures

\begin{tabular}{|c|c|c|c|c|c|}
\hline & \multicolumn{3}{|c|}{ choline equivalents in assay $(\mu \mathrm{M})$} & \multicolumn{2}{|c|}{$\begin{array}{l}\text { multivalent effect } \\
\text { ( } n \text {-fold, referred to choline chlor- } \\
\text { ide) }\end{array}$} \\
\hline & choline chloride & g5-cho PPI dendrimer ${ }^{35}$ & BTA-Chol $_{3}$ & g5-cho & BTA-Chol ${ }_{3}$ \\
\hline FA titration half-point & $2000 \pm 280$ & $5.0 \pm 0.2$ & $0.8 \pm 0.1$ & 400 & 2500 \\
\hline minimal concentration for bacterial chaining & 25,000 & 64 & 15 & 390 & 1667 \\
\hline
\end{tabular}



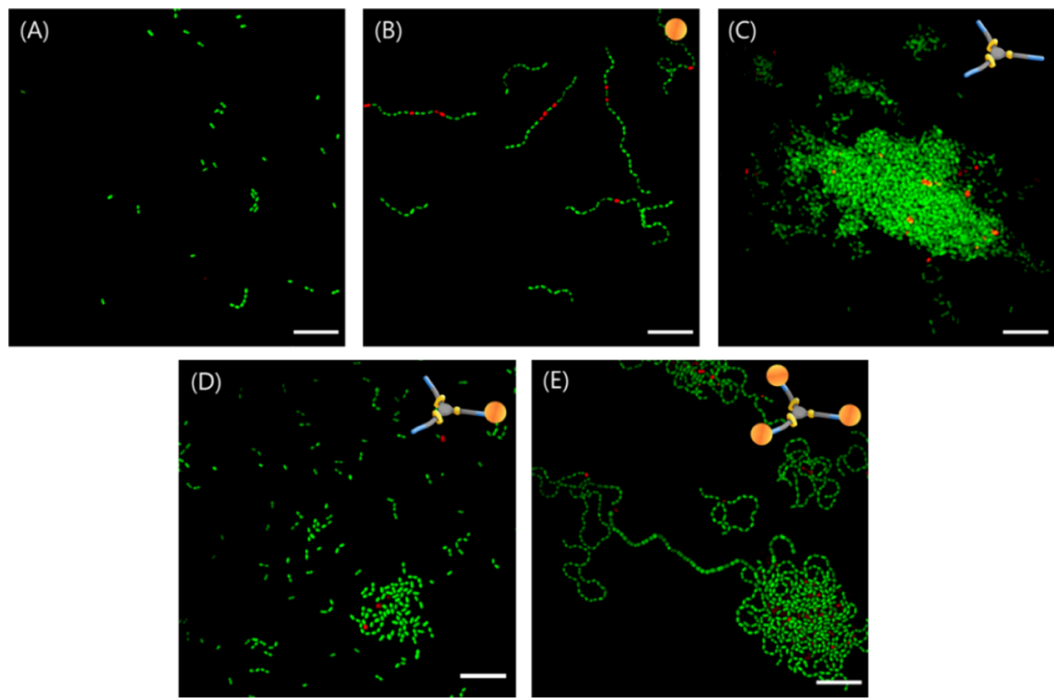

Figure 7. Effect of choline and BTA copolymers on S. pneumoniae growth. Confocal microscopy images of cultures of S. pneumoniae after 2 h of incubation in the (A) absence or in the presence of (B) $50 \mathrm{mM}$ choline chloride, (C) $50 \mu \mathrm{M}$ BTA-(OH) $)_{3}$, (D) $50 \mu \mathrm{M}$ BTA-Chol 1:9, and (E) $50 \mu \mathrm{M}$ BTA-Chol $_{3}$ 1:9. The scale bar represents $10 \mu \mathrm{m}$. Bacterial cultures are stained with BacLight.

(Figure S22). All these results are in accordance with the FA experiments on the binding of the fibers to the C-LytA CBM in the C-LytA-GFP fusion protein (Figure 5), as only in the presence of tri-substituted BTA monomers (BTA-Chol ${ }_{3}$ ), the fibers showed an efficient binding activity to CBPs.

In an attempt to unveil and quantify the multivalent effects of the BTA-Chol $_{3}$ 1:9 fibers in this biological context, we calculated the minimum concentration of choline equivalents able to induce a bacterial chaining phenotype in planktonic cultures and compared the results with free choline (Figure S21A) and with those previously reported for the g5-cho PPI dendrimer. $^{35}$ The data in Table 1 confirm that cholinefunctionalized BTA fibers display a higher "biological multivalency" value than that of the dendrimer ( 2000 vs $\sim 400$ respectively). This observation suggests that dynamic polymers may be more effective than static ones in molecular recognition processes. Most polydentate antimicrobial polymers possess a static nature in the sense that the bioactive ligands are attached to the polymer backbone via a flexible linker, but in a confined position within the polymer sequence. Therefore, a portion of the ligands may not be able to access their target. On the contrary, the dynamic nature of BTA polymers allows the rearrangement of the ligands along the fiber, thus enhancing the chance of binding with the target. In addition, the relative multivalency values for both the g5-cho PPI dendrimer and the BTA-Chol ${ }_{3}$ 1:9 fibers are of a comparable order of magnitude in both types of experiments (Table 1). It is remarkable that similar multivalency values were obtained from such different experimental approaches, that is, a complex physiological outcome from the inhibition of a set of enzymes in the microbiological experiment, and the direct binding between the structures and a protein.

It is also noteworthy that although BTA-Chol $_{3}$ 1:9 demonstrated a higher capacity to induce cell chaining compared to free choline or the g5-cho dendrimer, the fibers could not prevent autolysis to occur at the end of the stationary phase (Figure S21B). It should be pointed out that the different pneumococcal CBPs display a diverse range of affinities for choline. ${ }^{33}$ Therefore, the choline-decorated fibers could be very efficient in inhibiting the LytB $\mathrm{N}$-acetylglucosaminidase, a CBP that is mainly involved in daughter cell separation, leading to cell chains. However, the observation that autolysis still occurs indicates that the fibers are less proficient in inhibiting the LytA autolysin. $^{28}$ This behavior of formation of cell chains but no prevention of autolysis can also be seen in pneumococcal mutant strains lacking the LytB enzyme. ${ }^{50}$ This lack of inhibition of LytA is, however, in an apparent contradiction with the FA assays using C-LytA-GFP (Figure 5 and Table 1). A possible explanation for this discrepancy might be that the binding of the BTA-Chol ${ }_{3}$ monomers to the CBPs occurs in a slower time scale than the binding of a small ligand such as choline, possibly due to steric hindrance, diffusional impediments, and/or for the need or dynamic reorganization of the monomers within the fiber. Then, while LytB is permanently located on the cell surface and therefore has sufficient time to interact with the fibers, the release of LytA from the cytoplasm is triggered in a much narrower period (Figure S21). Therefore, the cell wall hydrolysis by LytA could start from the lower, more buried, layers of peptidoglycan prior to any binding interaction with the BTA fiber, which is located extracellularly. Nevertheless, the potential of BTA-Chol ${ }_{3} /$ BTA- $(\mathrm{OH})_{3}$ supramolecular copolymers as antipneumococcal agents is very promising since the induction of cell chains and aggregates is known to drastically decrease the infectivity of the cells and to promote phagocytosis by macrophages. Thus, the supramolecular copolymers might constitute the basis of a novel family of non-lytic antimicrobials that foster the protection by the immune system of the infected individual. $^{32-34}$

\section{CONCLUSIONS}

In an attempt to emulate the function of a biological macromolecular structure such as the choline-containing cell wall of the respiratory pathogen S. pneumoniae, we designed and synthesized a new set of BTA-based supramolecular polymers containing monomers that bear one or three functional groups at the outer periphery. The functionalized polymers were designed to behave as efficient competitors with the cell wall for the binding of pneumococcal CBPs. The functional groups consisted of hydrophilic and charged (choline) or hydrophobic (atropine) moieties. Incorporation of choline or atropine 
prevented the formation of supramolecular homopolymers, and small micellar aggregates were formed instead. The introduction of three atropine moieties even reduced the water solubility such that large precipitates were formed. This shows that the incorporation of functionality can greatly alter the behavior of BTA-based assemblies in water. Gratifyingly, by co-assembling choline-functionalized BTA-Chol and BTA-Chol ${ }_{3}$ with BTA$(\mathrm{OH})_{3}$ monomers, supramolecular copolymers were obtained. Depending on the monomer structure, the capability to copolymerize was slightly altered. BTA- $\mathbf{C h o l}_{3}$ displays more charged groups, has more repulsive interactions, and can be incorporated into supramolecular polymers at lower ratios than BTA-Chol. The H/D exchange of BTA- $\mathbf{C h o l}_{3}$ slowed down upon copolymerization with BTA- $(\mathbf{O H})_{3}$, indicating that the functional monomers are indeed incorporated into the supramolecular copolymer. Both BTA- $(\mathbf{O H})_{3}$ and BTA-Chol ${ }_{3}$ monomers remained dynamic within the supramolecular copolymer. Biophysical assays showed that BTA-Chol ${ }_{3}$ 1:9 copolymers were able to bind to CBPs in vitro with $\sim 2500$ - and $\sim 400$-fold enhanced binding affinity compared to free choline and a choline-derivatized generation-5 PPI dendrimer, respectively, due to a multivalent presentation of choline on the supramolecular copolymers. Such a higher binding efficiency was also reflected in microbiological experiments, which showed that BTA-Chol ${ }_{3}$ 1:9 copolymers were able to induce similar cell chaining effects as free choline as a result of CBP inhibition, but again with a 2000 -fold enhanced binding affinity and also outperforming previously studied dendrimer systems. As the conversion of the typical diplococcal shape of S. pneumoniae into long chains and cell aggregates has been described to have a deleterious effect on bacterial infectivity while promoting its phagocytosis by the host, our results show the potential of BTA supramolecular copolymers as a new scaffold for the design of novel antimicrobials.

\section{ASSOCIATED CONTENT}

\section{SI Supporting Information}

The Supporting Information is available free of charge at https://pubs.acs.org/doi/10.1021/acs.biomac.1c01293.

Additional synthetic procedures, NMR spectra of final compounds, HDX-MS data analysis, fit procedures of sigmoidal curves, calculation of the multivalent effect, additional cryo-TEM images, UV-vis spectra, and in vitro results (PDF)

\section{AUTHOR INFORMATION}

\section{Corresponding Authors}

Jesús M. Sanz - Centro de Investigaciones Biológicas Margarita Salas, Consejo Superior de Investigaciones Científicas, 28040 Madrid, Spain; Centro de Investigación Biomédica en Red de Enfermedades Respiratorias (CIBERES), 28029 Madrid, Spain; Email: jmsanz@cib.csic.es

E. W. Meijer - Laboratory of Macromolecular and Organic Chemistry, Eindhoven University of Technology, 5600 MB Eindhoven, The Netherlands; Institute for Complex Molecular Systems, Eindhoven University of Technology, $5600 \mathrm{MB}$ Eindhoven, The Netherlands; (1) orcid.org/0000-0003-41267492; Email: e.w.meijer@tue.nl

\section{Authors}

Marle E. J. Vleugels - Laboratory of Macromolecular and Organic Chemistry, Eindhoven University of Technology, 5600
MB Eindhoven, The Netherlands; Institute for Complex Molecular Systems, Eindhoven University of Technology, 5600 MB Eindhoven, The Netherlands; (1) orcid.org/0000-00026686-3568

Silvia Varela-Aramburu - Laboratory of Macromolecular and Organic Chemistry, Eindhoven University of Technology, 5600 MB Eindhoven, The Netherlands; Institute for Complex Molecular Systems, Eindhoven University of Technology, 5600 $M B$ Eindhoven, The Netherlands

Bas F. M. de Waal - Laboratory of Macromolecular and Organic Chemistry, Eindhoven University of Technology, 5600 MB Eindhoven, The Netherlands; Institute for Complex Molecular Systems, Eindhoven University of Technology, 5600 $M B$ Eindhoven, The Netherlands

Sandra M. C. Schoenmakers - Laboratory of Macromolecular and Organic Chemistry, Eindhoven University of Technology, 5600 MB Eindhoven, The Netherlands; Institute for Complex Molecular Systems, Eindhoven University of Technology, 5600 $M B$ Eindhoven, The Netherlands

Beatriz Maestro - Centro de Investigaciones Biológicas Margarita Salas, Consejo Superior de Investigaciones Científicas, 28040 Madrid, Spain; 이이.org/0000-00015317-650X

Anja R. A. Palmans - Laboratory of Macromolecular and Organic Chemistry, Eindhoven University of Technology, 5600 MB Eindhoven, The Netherlands; Institute for Complex Molecular Systems, Eindhoven University of Technology, 5600 MB Eindhoven, The Netherlands; (1) orcid.org/0000-00027201-1548

Complete contact information is available at: https://pubs.acs.org/10.1021/acs.biomac.1c01293

\section{Author Contributions}

M.E.J.V., A.R.A.P., E.W.M., and J.M.S conceived and designed the research. M.E.J.V., S.V-A., B.F.M.d.W. S.M.C.S., and B.M. performed all the experiments. The manuscript was written through contributions of all authors. All authors have given approval to the final version of the manuscript.

\section{Notes}

The authors declare no competing financial interest.

\section{ACKNOWLEDGMENTS}

We gratefully acknowledge A.J.H. Spiering for synthetic support. The authors acknowledge the financial support from the Dutch Ministry of Education, Culture and Science (Gravity program 024.001.035), the ERC Advanced Grant (SYNMAT-788618), the Gravitation Program "Materials Driven Regeneration", funded by the Netherlands Organization for Scientific Research (024.003.013), and the Agencia Estatal de Investigacion AEI/ FEDER-EU-10.13039/501100011033- and Ministerio de Ciencia e Innovación, Spain (PID2019-105126RB-I00). The CIBER de Enfermedades Respiratorias (CIBERES) is an initiative of the Spanish Instituto de Salud Carlos III.

\section{REFERENCES}

(1) Krieg, E.; Bastings, M. M. C.; Besenius, P.; Rybtchinski, B. Supramolecular Polymers in Aqueous Media. Chem. Rev. 2016, 116, 2414-2477.

(2) Fowler, D. M.; Koulov, A. V.; Balch, W. E.; Kelly, J. W. Functional Amyloid - from Bacteria to Humans. Trends Biochem. Sci. 2007, 32, 217-224.

(3) Mankar, S.; Anoop, A.; Sen, S.; Maji, S. K. Nanomaterials: Amyloids Reflect Their Brighter Side. Nano Rev. 2011, 2, 6032. 
(4) Aida, T.; Meijer, E. W.; Stupp, S. I. Functional Supramolecular Polymers. Science 2012, 335, 813-817.

(5) Webber, M. J.; Appel, E. A.; Meijer, E. W.; Langer, R. Supramolecular Biomaterials. Nat. Mater. 2015, 15, 13-26.

(6) Hendricks, M. P.; Sato, K.; Palmer, L. C.; Stupp, S. I. Supramolecular Assembly of Peptide Amphiphiles. Acc. Chem. Res. 2017, 50, 2440-2448.

(7) Mata, A.; Geng, Y.; Henrikson, K. J.; Aparicio, C.; Stock, S. R.; Satcher, R. L.; Stupp, S. I. Bone Regeneration Mediated by Biomimetic Mineralization of a Nanofiber Matrix. Biomaterials 2010, 31, 60046012.

(8) Lee, S. S.; Fyrner, T.; Chen, F.; Álvarez, Z.; Sleep, E.; Chun, D. S.; Weiner, J. A.; Cook, R. W.; Freshman, R. D.; Schallmo, M. S.; Katchko, K. M.; Schneider, A. D.; Smith, J. T.; Yun, C.; Singh, G.; Hashmi, S. Z.; McClendon, M. T.; Yu, Z.; Stock, S. R.; Hsu, W. K.; Hsu, E. L.; Stupp, S. I. Sulfated Glycopeptide Nanostructures for Multipotent Protein Activation. Nat. Nanotechnol. 2017, 12, 821-829.

(9) Dankers, P. Y. W.; Hermans, T. M.; Baughman, T. W.; Kamikawa, Y.; Kieltyka, R. E.; Bastings, M. M. C.; Janssen, H. M.; Sommerdijk, N. A. J. M.; Larsen, A.; Van Luyn, M. J. A.; Bosman, A. W.; Popa, E. R.; Fytas, G.; Meijer, E. W. Hierarchical Formation of Supramolecular Transient Networks in Water: A Modular Injectable Delivery System. Adv. Mater. 2012, 24, 2703-2709.

(10) Pape, A.; Bastings, M.; Kieltyka, R.; Wyss, H.; Voets, I.; Meijer, E.; Dankers, P. Mesoscale Characterization of Supramolecular Transient Networks Using SAXS and Rheology. Int. J. Mol. Sci. 2014, 15, 1096-1111.

(11) Dankers, P. Y. W.; Harmsen, M. C.; Brouwer, L. A.; Van Luyn, M. J. A.; Meijer, E. W. A Modular and Supramolecular Approach to Bioactive Scaffolds for Tissue Engineering. Nat. Mater. 2005, 4, 568574.

(12) Bakker, M. H.; Kieltyka, R. E.; Albertazzi, L.; Dankers, P. Y. W. Modular Supramolecular Ureidopyrimidinone Polymer Carriers for Intracellular Delivery. RSC Adv. 2016, 6, 110600-110603.

(13) Leenders, C. M. A.; Albertazzi, L.; Mes, T.; Koenigs, M. M. E.; Palmans, A. R. A.; Meijer, E. W. Supramolecular Polymerization in Water Harnessing Both Hydrophobic Effects and Hydrogen Bond Formation. Chem. Commun. 2013, 49, 1963-1965.

(14) Leenders, C. M. A.; Baker, M. B.; Pijpers, I. A. B.; Lafleur, R. P. M.; Albertazzi, L.; Palmans, A. R. A.; Meijer, E. W. Supramolecular Polymerisation in Water; Elucidating the Role of Hydrophobic and Hydrogen-Bond Interactions. Soft Matter 2016, 12, 2887-2893.

(15) Lafleur, R. P. M.; Herziger, S.; Schoenmakers, S. M. C.; Keizer, A. D. A.; Jahzerah, J.; Thota, B. N. S.; Su, L.; Bomans, P. H. H.; Sommerdijk, N. A. J. M.; Palmans, A. R. A.; Haag, R.; Friedrich, H.; Böttcher, C.; Meijer, E. W. Supramolecular Double Helices from Small C3-Symmetrical Molecules Aggregated in Water. J. Am. Chem. Soc. 2020, 142, 17644-17652.

(16) Lou, X.; Lafleur, R. P. M.; Leenders, C. M. A.; Schoenmakers, S. M. C.; Matsumoto, N. M.; Baker, M. B.; Van Dongen, J. L. J.; Palmans, A. R. A.; Meijer, E. W. Dynamic Diversity of Synthetic Supramolecular Polymers in Water as Revealed by Hydrogen/Deuterium Exchange. Nat. Commun. 2017, 8, 15420.

(17) Baker, M. B.; Gosens, R. P. J.; Albertazzi, L.; Matsumoto, N. M.; Palmans, A. R. A.; Meijer, E. W. Exposing Differences in Monomer Exchange Rates of Multicomponent Supramolecular Polymers in Water. ChemBioChem 2016, 17, 207-213.

(18) Albertazzi, L.; Martinez-Veracoechea, F. J.; Leenders, C. M. A.; Voets, I. K.; Frenkel, D.; Meijer, E. W. Spatiotemporal Control and Superselectivity in Supramolecular Polymers Using Multivalency. Proc. Natl. Acad. Sci. U.S.A. 2013, 110, 12203-12208.

(19) Leenders, C. M. A.; Jansen, G.; Frissen, M. M. M.; Lafleur, R. P. M.; Voets, I. K.; Palmans, A. R. A.; Meijer, E. W. Monosaccharides as Versatile Units for Water-Soluble Supramolecular Polymers. Chem.Eur. J. 2016, 22, 4608-4615.

(20) Schoenmakers, S. M. C.; Leenders, C. M. A.; Lafleur, R. P. M.; Lou, X.; Meijer, E. W.; Pavan, G. M.; Palmans, A. R. A. Impact of the Water-Compatible Periphery on the Dynamic and Structural Properties of Benzene-1,3,5-Tricarboxamide Based Amphiphiles. Chem. Commun. 2018, 54, 11128-11131.

(21) Hendrikse, S. I. S.; Su, L.; Hogervorst, T. P.; Lafleur, R. P. M.; Lou, X.; Van Der Marel, G. A.; Codee, J. D. C.; Meijer, E. W. Elucidating the Ordering in Self-Assembled Glycocalyx Mimicking Supramolecular Copolymers in Water. J. Am. Chem. Soc. 2019, 141, 13877-13886.

(22) Morgese, G.; Waal, B. F. M.; Varela-Aramburu, S.; Palmans, A. R. A.; Albertazzi, L.; Meijer, E. W. Anchoring Supramolecular Polymers to Human Red Blood Cells by Combining Dynamic Covalent and NonCovalent Chemistries. Angew. Chem., Int. Ed. 2020, 132, 17382-17386.

(23) Kiessling, L.; Gestwicki, J. E.; Strong, L. E. Synthetic Multivalent Ligands in the Exploration of Cell-Surface Interactions. Curr. Opin. Chem. Biol. 2000, 4, 696-703.

(24) Zhu, X.; Ge, Y.; Wu, T.; Zhao, K.; Chen, Y.; Wu, B.; Zhu, F.; Zhu, B.; Cui, L. Co-Infection with Respiratory Pathogens among COVID2019 Cases. Virus Res. 2020, 285, 198005.

(25) US Department of Health and Human Services; CDC. Antibiotic Resistance Threats in the United States. http://dx.doi.org/10.15620/ cdc:82532 (accessed July 19, 2021).

(26) Maestro, B.; Sanz, J. Novel Approaches To Fight Streptococcus pneumoniae. Recent Pat. Anti-Infect. Drug Discovery 2008, 2, 188-196.

(27) Domenech, M.; García, E.; Moscoso, M. Biofilm Formation in Streptococcus pneumoniae. Microb. Biotechnol. 2012, 5, 455-465.

(28) Maestro, B.; Sanz, J. Choline Binding Proteins from Streptococcus pneumoniae: A Dual Role as Enzybiotics and Targets for the Design of New Antimicrobials. Antibiotics 2016, 5, 21.

(29) Briese, T.; Hakenbeck, R. Interaction of the Pneumococcal Amidase with Lipoteichoic Acid and Choline. Eur. J. Biochem. 1985, 146, 417-427.

(30) Sanz, J. M.; López, R.; García, J. L. Structural Requirements of Choline Derivatives for "conversion" of Pneumococcal Amidase A New Single-Step Procedure for Purification of This Autolysin. FEBS Lett. 1988, 232, 308-312.

(31) Maestro, B.; González, A.; García, P.; Sanz, J. M. Inhibition of Pneumococcal Choline-Binding Proteins and Cell Growth by Esters of Bicyclic Amines. FEBS J. 2007, 274, 364-376.

(32) Dalia, A. B.; Weiser, J. N. Minimization of Bacterial Size Allows for Complement Evasion and Is Overcome by the Agglutinating Effect of Antibody. Cell Host Microbe 2011, 10, 486-496.

(33) Roig-Molina, E.; Sánchez-Angulo, M.; Seele, J.; García-Asencio, F.; Nau, R.; Sanz, J. M.; Maestro, B. Searching for Antipneumococcal Targets: Choline-Binding Modules as Phagocytosis Enhancers. ACS Infect. Dis. 2020, 6, 954-974.

(34) Ribes, S.; Riegelmann, J.; Redlich, S.; Maestro, B.; De Waal, B.; Meijer, E. W.; Sanz, J. M.; Nau, R. Multivalent Choline Dendrimers Increase Phagocytosis of Streptococcus pneumoniae R6 by Microglial Cells. Chemotherapy 2013, 59, 138-142.

(35) Hernández-Rocamora, V. M.; Maestro, B.; de Waal, B.; Morales, M.; García, P.; Meijer, E. W.; Merkx, M.; Sanz, J. M. Multivalent Choline Dendrimers as Potent Inhibitors of Pneumococcal Cell-Wall Hydrolysis. Angew. Chem., Int. Ed. 2009, 48, 948-951.

(36) De Gracia Retamosa, M.; Díez-Martínez, R.; Maestro, B.; GarcíaFernández, E.; de Waal, B.; Meijer, E. W.; García, P.; Sanz, J. M. Aromatic Esters of Bicyclic Amines as Antimicrobials against Streptococcus pneumoniae. Angew. Chem., Int. Ed. 2015, 54, 1367313677.

(37) Kunishima, M.; Kawachi, C.; Monta, J.; Terao, K.; Iwasaki, F.; Tani, S. 4-(4,6-Dimethoxy-1,3,5-Triazin-2-Yl)-4-Methylmorpholinium Chloride: An Efficient Condensing Agent Leading to the Formation of Amides and Esters. Tetrahedron 1999, 55, 13159-13170.

(38) Roosma, J.; Mes, T.; Leclère, P.; Palmans, A. R. A.; Meijer, E. W. Supramolecular Materials from Benzene-1,3,5-Tricarboxamide-Based Nanorods. J. Am. Chem. Soc. 2008, 130, 1120-1121.

(39) Sánchez-Puelles, J.; Sanz, J. M.; Garcia, J.; Garcia, E. Cloning and Expression of Gene Fragments Encoding the Choline-Binding Domain of Pneumococcal Murein Hydrolases. Gene 1990, 89, 69-75.

(40) Fernández-Tornero, C.; López, R.; García, E.; Giménez-Gallego, G.; Romero, A. A Novel Solenoid Fold in the Cell Wall Anchoring 
Domain of the Pneumococcal Virulence Factor LytA. Nat. Struct. Mol. Biol. 2001, 8, 1020-1024.

(41) Grosdidier, A.; Zoete, V.; Michielin, O. SwissDock, a ProteinSmall Molecule Docking Web Service Based on EADock DSS. Nucleic Acids Res. 2011, 39, W270.

(42) Grosdidier, A.; Zoete, V.; Michielin, O. Fast Docking Using the CHARMM Force Field with EADock DSS. J. Comput. Chem. 2011, 32, 2149-2159.

(43) Hoskins, J.; Alborn, W. E.; Arnold, J.; Blaszczak, L. C.; Burgett, S.; Dehoff, B. S.; Estrem, S. T.; Fritz, L.; Fu, D.-J.; Fuller, W.; Geringer, C.; Gilmour, R.; Glass, J. S.; Khoja, H.; Kraft, A. R.; Lagace, R. E.; LeBlanc, D. J.; Lee, L. N.; Lefkowitz, E. J.; Lu, J.; Matsushima, P.; McAhren, S. M.; McHenney, M.; McLeaster, K.; Mundy, C. W.; Nicas, T. I.; Norris, F. H.; O'Gara, M.; Peery, R. B.; Robertson, G. T.; Rockey, P.; Sun, P.M.; Winkler, M. E.; Yang, Y.; Young-Bellido, M.; Zhao, G.; Zook, C. A.; Baltz, R. H.; Jaskunas, S. R.; Rosteck, P. R.; Skatrud, P. L.; Glass, J. I. Genome of the Bacterium Streptococcus pneumoniae Strain R6. J. Bacteriol. 2001, 183, 5709-5717.

(44) Lacks, S.; Hotchkiss, R. D. A Study of the Genetic Material Determining an Enzyme Activity in Pneumococcus. Biochim. Biophys. Acta 1960, 39, 508-518.

(45) Thota, B. N. S.; Lou, X.; Bochicchio, D.; Paffen, T. F. E.; Lafleur, R. P. M.; van Dongen, J. L. J.; Ehrmann, S.; Haag, R.; Pavan, G. M.; Palmans, A. R. A.; Meijer, E. W. Supramolecular Copolymerization as a Strategy to Control the Stability of Self-Assembled Nanofibers. Angew. Chem., Int. Ed. 2018, 57, 6843-6847.

(46) Lou, X.; Schoenmakers, S. M. C.; Dongen, J. L. J.; Garcia-Iglesias, M.; Casellas, N. M.; Fernández-Castaño Romera, M.; Sijbesma, R. P.; Meijer, E. W.; Palmans, A. R. A. Elucidating Dynamic Behavior of Synthetic Supramolecular Polymers in Water by Hydrogen/Deuterium Exchange Mass Spectrometry. J. Polym. Sci. 2021, 59, 1151-1161.

(47) Lafleur, R. P. M.; Schoenmakers, S. M. C.; Madhikar, P.; Bochicchio, D.; Baumeier, B.; Palmans, A. R. A.; Pavan, G. M.; Meijer, E. W. Insights into the Kinetics of Supramolecular Comonomer Incorporation in Water. Macromolecules 2019, 52, 3049-3055.

(48) Usobiaga, P.; Medrano, F. J.; Gasset, M.; García, J. L.; Saiz, J. L.; Rivas, G.; Laynez, J.; Menéndez, M. Structural Organization of the Major Autolysin from Streptococcus pneumoniae. J. Biol. Chem. 1996, 271, 6832-6838.

(49) Howard, L. V.; Gooder, H. Specificity of the Autolysin of Streptococcus (Diplococcus) pneumoniae. J. Bacteriol. 1974, 117, 796804.

(50) De Las Rivas, B.; García, J. L.; López, R.; García, P. Purification and Polar Localization of Pneumococcal LytB, a Putative Endo- $\beta$-NAcetylglucosaminidase: The Chain-Dispersing Murein Hydrolase. J. Bacteriol. 2002, 184, 4988-5000. 